\title{
The experimental application of steam explosion-pre-treated flaxseed meal with hypoglycaemic and lipid-lowering functions in rats on a high-fat-sugar diet
}

\author{
G.W. Yu ${ }^{1,2,3}$, T.T. Guo ${ }^{1,2,3}$ X. Zhou ${ }^{1,2,3}$, Q.D. Huang ${ }^{1,2,3 *}$ and X.W. Shi ${ }^{1,2,3 *}$ \\ ${ }^{1}$ Oil Crops Research Institute, Chinese Academy of Agricultural Sciences, Wuhan, China; ${ }^{2}$ Hubei Key Laboratory of Lipid \\ Chemistry and Nutrition, Wuhan, China; ${ }^{3}$ Oil Crops and Lipids Process Technology National E Local Joint Engineering \\ Laboratory, Wuhan, China
}

Corresponding Author: Q.D. Huang, X.W. Shi; m18202747986@163.com

Received: 28 January 2020 / Accepted: 25 March 2020 / Published: 20 May 2020. (C) 2020 Codon Publications

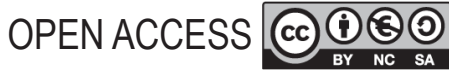
RESEARCH ARTICLE

\begin{abstract}
With the increase in populations with suboptimal health and chronic diseases, consumer demands for health foods, especially food products with health functions. The flaxseed was a complex mixture mainly consisting of flaxseed oil, flaxseed protein, dietary fibre (including cellulose and flaxseed gum), carbohydrates and secoisolariciresinol diglucoside (SDG). To investigate the effects of expanded flaxseed meal (EFM) intervention on the hypoglycaemic and lipid-lowering functions of rats on a high-fat, high-sugar (HFHS) diet, EFM was fed to six groups $(z=8)$ of Sprague-Dawley (SD) rats: normal control, HFHS model, positive treatment control, and low-, medium-, and high-dose EFM (5\%,10\% and 20\% mass fraction EFM, respectively) groups. The results (8 weeks later) were as follows: (1) medium- and high-dose EFM achieved similar inhibitive effects on blood glucose levels compared with positive treatment $(5.94 \pm 0.32 \mathrm{mmol} / \mathrm{L})$; (2) all EFM doses significantly reduced total cholesterol, triglyceride and low-density lipoprotein cholesterol levels in the blood plasma of SD rats and (3) EFM significantly increased glutathione peroxidase, superoxide dismutase and catalase activities, increased glutathione levels, and reduced the malondialdehyde content in the plasma of SD rats $(P<0.05)$. Therefore, EFM mitigated the adverse effects produced by HFHS diet on physiological functions, effectively regulated blood glucose and lipid metabolism, enhanced plasma antioxidant capacity and alleviated oxidative stress in SD rats with a certain dose-effect relationship among the EFM dose groups. Hepatic histological morphologies indicated that EFM also improved the size and density of lipid droplets in the liver of the rats and inhibited the onset of fatty liver. These results suggest that EFM is a potentially useful raw material in the development of blood glucose and lipid-lowering food products.
\end{abstract}

Keywords: expanded flaxseed meal, SD rats, hypoglycaemic activity, hypolipidemic activity, oxidative stress, hepatic histological morphology

\section{Introduction}

Noteworthily, an increasing amount of attention has been diverted to the utilisation of 'medicine-food homology' foods (medicine-food homology refers to the concept that foods are drugs and there is no absolute demarcation between them) (Chu et al., 2016; Song and Jiang, 2017). Flaxseed mainly consists of fat, protein and dietary fibre, but also contains other components, such as carbohydrates, flaxseed gum and flax lignans (Bekhit et al., 2018). According to the health-based guidance values (HBGVs) for cyanides established by the Joint FAO/WHO Expert Committee on Food Additives (JECFA), the risk of cyanides originating from cooked flaxseed to human health is extremely low (Cressey and Reeve, 2019). Processing methods, such as heating, microwaving and cooking, can reduce cyanogenic glycoside contents in flaxseed 
and its products, and enhance the safety of flaxseed (Cao et al., 2016; Yang et al., 2008; Zhao et al., 2019). In certain regions of China, such as Gansu, Inner Mongolia and Ningxia, flaxseed (meal) is used as an ingredient in processed flour products or as a condiment for dipping after grinding and flavouring with salt (Edrosolam and Shand, 2014; Ganorkar et al., 2016).

Studies and clinical trials have proven that flaxseed has several beneficial effects, including cholesterol reduction, lowering of cardiac load and promotion of cellular health and brain development (Chirino-Galindo et al., 2017; Han et al., 2015; Liu and Ma, 2014). Han et al. (2018) demonstrated that the partial replacement of lard with flaxseed oil significantly improved atherosclerosis, dyslipidaemia, oxidative stress and inflammation in mice, indicating that flaxseed oil plays an important role in the improvement of cardiovascular health and is a promising functional food. Wang et al. (2015) showed that secoisolariciresinol diglucoside (SDG), a lignan abundant in flaxseed, could reduce the fasting blood glucose, insulin and free fatty acid levels in mice on a high-fat diet by feeding obese mice with SDG solutions of different concentrations. In addition, it was deduced that SDG may increase the ability of diet-induced obese mice to control blood glucose levels within a certain range by enhancing insulin signal transduction and sensitivity. Luo et al. (2018) investigated the blood glucose control and anti-obesity effects of flaxseed gum and its influence on gut microbiota by feeding mice with different doses of flaxseed gum, measuring biochemical indicators, such as body fat content and short-chain fatty acid content, and analysing metagenome of the gut microbiota. Their results indicated that a flaxseed gum diet provided multiple beneficial effects, including the reduction of body weight, lowering of body fat content and total triglycerides, and reconstitution of the cecal microbiota of rats.

Under the influence of traditional eating habits and cultures, cooked flaxseed with highly concentrated fragrances is more favoured by consumers (Alberdicedeño et al., 2017). Notably, the pre-treatment processes currently used in the production of cooked flaxseed lead to a number of disadvantages, including the loss of micronutrient components, food safety risk indices that exceed the limitation standards and poor fragrances. Steam explosion technology is a physicochemical pre-treatment method that transforms thermal energy into mechanical energy and achieves the separation and structural changes of macromolecule substances in components through instantaneous pressure release and expansion under a high temperature and pressure environment (Li et al., 2019; Shi et al., 2019; Zhang et al., 2019). To date, studies of steam explosion technology to pre-treat fresh flaxseed and its effect on the nutritional and volatile components of flaxseed oil are researched in our laboratory. Compared with traditional high-temperature roasting and popular microwave pre-treatment techniques, steam explosion-pre-treated flaxseed shows such many unique advantages as high-quality concentrated fragrance characteristics, better taste and so on (dates are not shown). However, studies on the influence of the addition of expanded flaxseed meal (EFM) to the diet on body functions have rarely been reported. Therefore, in this study, EFM was fed to rats on a high-fat, high-sugar (HFHS) diet, and the physiological and biochemical indicators of the rats were measured. The influence of EFM on the blood glucose and lipid levels, and hepatic histological morphologies of the experimental animals, was evaluated, so as to determine if EFM possesses regulatory effects on certain aspects of suboptimal health and provide supporting fundamental data for the use of EFM in the development of medicine-food homology products.

\section{Materials and methods}

\section{Experimental materials}

All experimental protocols were approved by the Ethical Committee of the Faculty of Veterinary Medicine, Huazhong Agricultural University (permit number: SYXK-ER-2013-0044). The study was performed in strict accordance with the recommendations of the Guide for the Care and Use of Laboratory Animals in China. A total of 48 specific pathogen-free grade male SpragueDawley (SD) rats weighing 180-220 g were purchased from the Hubei Provincial Center of Disease Control and Prevention (Wuhan, China) and maintained in an isolated specific pathogen free (SPF) room with a constantly regulated temperature $\left(22 \pm 2{ }^{\circ} \mathrm{C}\right)$ under a 12:12-h light/ dark cycle. Yellow flaxseed with a large seed size was purchased from Jinchang City, Gansu Province, China; lard was prepared in our laboratory; AIN-93M feed (all percentages by weight: $62 \%$ corn starch, $14 \%$ casein, $10 \%$ cane sugar, $5 \%$ cellulose, $4 \%$ soybean oil, $3.5 \%$ multiple salts, $1 \%$ multiple vitamins, $0.3 \%$ DL-methionine, $0.2 \%$ choline chloride) was purchased from Medisciences Ltd. (Jiangsu, China); white sugar was purchased from Shanghai Sugar, Cigarette \& Wine (Group) Co., Ltd. (Shanghai, China); acarbose tablets were purchased from Bayer Health Care LLC. (Beijing, China); low-density lipoprotein cholesterol (LDL-C), high-density lipoprotein cholesterol (HDL-C), total triglyceride (TG), total cholesterol (TC) and glycated haemoglobin (HbA1c) assay kits were purchased from Beijing Leadman Biochemistry Co., Ltd. (China); hepatic glycogen assay kits were purchased from Nanjing Jiancheng Bioengineering Institute (Nanjing, China); and blood glucose test strips were purchased from Roche Pharmaceuticals. 


\section{Experimental methods}

\section{EFM preparation}

EFM was obtained after subjecting flaxseed to steam explosion pre-treatment and grinding which were, respectively, carried out on an XSS-QPD multifunctional air expander (KINHE Food Machinery Co., Ltd., Wuhan, China) and FW80 high-speed grinder (Taisite Instrument Co., Ltd., Tianjin, China). An appropriate amount of fresh flaxseed was weighed, and water was added for tempering to maintain the moisture content at $\sim 8 \%$. After moisture equilibration had been achieved, the flaxseed was placed in the chamber of an airflow puffing machine equipped with a thermostat. When the temperature had stabilised $\left(\sim 220{ }^{\circ} \mathrm{C}\right)$ and the set pressure $(1.2 \mathrm{MPa})$ had been attained within the chamber, the system was explosively depressurised to normal pressure within an extremely short period of time ( $\mathrm{Li}$ et al., 2019). Subsequently, the pre-treated flaxseed was ground in a mill and sieved through a 60 -mesh screen. A sample of the EFM was removed for component testing, and the remaining EFM was sealed and stored at $4{ }^{\circ} \mathrm{C}$ before further use.

\section{Grouping and processing of experimental animals}

Six groups were established, based on the diets indicated in parentheses (all percentages by weight): (1) normal control (AIN-93M feed); (2) HFHS model (65.5\% AIN93M feed, $12 \%$ lard, 20\% white sugar, $2 \%$ cholesterol, $0.5 \%$ sodium cholate); (3) low-dose EFM (95\% HFHS feed, 5\% EFM); (4) medium-dose EFM (90\% HFHS feed, 10\% EFM); (5) high-dose EFM (80\% HFHS feed, 20\% EFM) and (6) Positive control ( $0.15 \mathrm{~g}$ acarbose tablet per $\mathrm{kg}$ of feed) groups.

After acclimatisation with normal feed for 3 days, the SD rats were randomised into the six groups stated above (eight rats per group), and subjected to experimental feeding. Food intake was recorded daily and the rats were weighed twice weekly. After 8 weeks of experimental feeding, the rats were fasted overnight with normal access to water for $12 \mathrm{~h}$ and subsequently anaesthetised with $3 \%$ phenobarbital. Blood samples were withdrawn by orbital puncture, set aside for $1 \mathrm{~h}$ in Eppendorf tubes, and centrifuged for $10 \mathrm{~min}$ at 4000 $\mathrm{r} / \mathrm{min}$ for separation of the serum. After blood sample collection, the liver, kidney and spleen were removed from each rat, washed with chilled saline, patted dry with filter paper and weighed using an electronic balance to determine the wet weights of the organs. The separated serum and organs were stored at $-80^{\circ} \mathrm{C}$ prior to further testing.

\section{Measurement and calculation of physicochemical indicators and the physiological and biochemical indicators of experimental animals}

\section{Main components of EFM}

The moisture, ash, protein, fat, dietary fibre and cyanide contents of EFM were measured in accordance with the Chinese standards, GB 5009.3-2016, GB 5009.4-2016, GB 5009.5-2016, GB 5009.6-2016, GB 5009.88-2014 and GB 5009.36-2016, respectively. SDG content was measured in accordance with the method reported by Zang et al. (2017).

\section{Rate of body weight gain}

$Y=\frac{X_{2}-X_{1}}{X_{1}} \times 100 \%$

where $Y$ is the rate of body weight gain of an SD rat, and $X_{1}$ and $X_{2}$ are the initial and final body weights $(\mathrm{g})$.

\section{Organ coefficients}

$Y=\frac{X_{i}}{X_{2}} \times 100 \%$

where $Y$ is the organ coefficient of an SD rat, $X i$ is the wet weight of its organ, $i$, (g), and $X_{2}$ is its final body weight (g).

\section{Blood glucose and blood lipid levels}

Fasting blood glucose levels were measured using a blood glucose meter and blood glucose test strips blood glucose meter (Roche Pharmaceutical Co., Ltd., Shanghai, China).

HbA1c level, serum TC, TG, LDL-C, and HDL-C concentrations, the hepatic glycogen concentration in liver tissue and glutathione peroxidase (GSH-Px), superoxide dismutase (SOD), catalase (CAT), glutathione (GSH) and malondialdehyde (MDA) were measured in accordance with the instructions of the corresponding assay kits by using the automated HITACHI 3100 biochemical analyser (Ogino Biotechnology Co., Ltd., Shanghai, China) and enSpire plate reader (PerkinElmer, USA).

\section{Atherosclerosis-related indicators}

$\mathrm{AI}=\frac{\mathrm{TC}-\mathrm{HDLC}}{\mathrm{HDLC}}$

$\mathrm{AAI}=\frac{\mathrm{HDLC}}{\mathrm{TC}}$ 
Paraffin embedding and haematoxylin and eosin staining of liver sections

Liver sections of the SD rats were subjected to paraffin embedding and haematoxylin and eosin (H\&E) staining. Tissue sections were fixed in neutral formalin, dewatered, embedded in paraffin wax, sliced, stained with H\&E and mounted in neutral balsam. The liver tissue structures of the various groups were observed and photographed under the Olympus BX43 Biological Microscope (Puhe International Trade Co., Ltd., Shanghai, China). An image analysis system was used to analyse the H\&E staining results of the paraffin-embedded liver tissues, and changes in the size and density of lipid droplets in the tissue sections were observed microscopically.

\section{Data analyses}

Statistical analyses were performed using the SPSS program (SPSS 23.0 for Windows, SPSS Inc., Chicago, IL, USA). Duncan's test at the $5 \%$ significance level $(P<0.05)$ and one-way analysis of variance (ANOVA) were used to determine the significance differences.

\section{Results and discussion}

\section{Main components of EFM}

Component testing results indicated that EFM (expansion ratio and water solubility index (\%) were 1.62 and 48.89 , respectively) mainly consisted of the following (all percentages by weight): 1.9\% moisture, 2.9\% total ash, $29.4 \%$ dietary fibres, $40.7 \%$ fat, $23.1 \%$ protein, 32.8 $\mathrm{mg} / \mathrm{g}$ SDG and undetectable level of cyanogenic glycosides. In addition, the mass percentage of dietary fibre, which is regarded as a substance that produces a feeling of fullness, boosts peristalsis and controls energy absorption despite being indigestible and non-absorbable from the gastrointestinal tract (Wang et al., 2018), was as high as $40.1 \%$ in EFM. It was also found that EFM contained $32.8 \mathrm{mg} / \mathrm{g}$ of SDG, which is a physiologically active component.

\section{Body weight gain and organ coefficients of SD rats}

Table 2 shows the body weight gain and organ coefficients of the SD rats after 8 weeks of experimental feeding. The high-dose EFM group had the highest rate of body weight gain, followed by the medium-dose EFM and normal control groups, which had similar rates of body weight gain. A significantly lower rate of body weight gain was observed in the positive control group, which may be attributed to reduced food intake caused by the poor adaptation of experimental animals to the texture of the HFHS feed and the effects of acarbose (Wang et al., 2018). The addition of EFM significantly enhanced the palatability of the HFHS feed; consequently, there were no significant differences in the rate of body weight gain of the normal control group and the various EFM dose groups $(P>0.05)$. From Table 2 , it can be seen that rats on the HFHS diet had a significantly higher liver coefficient compared with the normal

Table 1. Main components of expanded flaxseed meal.

\begin{tabular}{cccccccc} 
Group & $\begin{array}{c}\text { Moisture } \\
(\%)\end{array}$ & $\begin{array}{c}\text { Total ash } \\
(\%)\end{array}$ & $\begin{array}{c}\text { Dietary fibre } \\
(\%)\end{array}$ & $\begin{array}{c}\text { Fat } \\
(\%)\end{array}$ & $\begin{array}{c}\text { Protein } \\
(\%)\end{array}$ & $\begin{array}{c}\text { SDG } \\
(\mathbf{m g} / \mathbf{g})\end{array}$ & $\begin{array}{c}\text { Cyanogenic } \\
\text { glycosides } \\
(\mathrm{mg} / \mathbf{k g})\end{array}$ \\
\hline EFM & $1.9 \pm 0.0$ & $2.9 \pm 0.1$ & $29.4 \pm 0.2$ & $40.7 \pm 0.6$ & $23.1 \pm 0.5$ & $32.8 \pm 0.3$ & Undetectable \\
\hline
\end{tabular}

EFM, expanded flaxseed meal; SDG, secoisolariciresinol diglucoside.

Table 2. Body weight gain and organ coefficients of Sprague-Dawley rats.

\begin{tabular}{lcccccc} 
Group & $\begin{array}{c}\text { No. of } \\
\text { deaths }\end{array}$ & $\begin{array}{c}\text { Body weight } \\
\text { gain (g) }\end{array}$ & $\begin{array}{c}\text { Rate of body } \\
\text { weight gain (\%) }\end{array}$ & $\begin{array}{c}\text { Liver } \\
\text { coefficient (\%) }\end{array}$ & $\begin{array}{c}\text { Kidney } \\
\text { coefficient (\%) }\end{array}$ & $\begin{array}{c}\text { Spleen } \\
\text { coefficient (\%) }\end{array}$ \\
\hline Normal control & 0 & $295.8 \pm 12.58^{\mathrm{a}}$ & $137.53 \pm 5.50^{\mathrm{a}}$ & $3.39 \pm 0.27^{\mathrm{c}}$ & $0.55 \pm 0.10^{\mathrm{a}}$ & $0.16 \pm 0.05^{\mathrm{a}}$ \\
HFHS model & 0 & $228.0 \pm 11.52^{\mathrm{b}}$ & $102.93 \pm 5.20^{\mathrm{b}}$ & $5.49 \pm 0.25^{\mathrm{a}}$ & $0.57 \pm 0.08^{\mathrm{a}}$ & $0.26 \pm 0.08^{\mathrm{a}}$ \\
Positive control & 0 & $193.8 \pm 13.26^{\mathrm{c}}$ & $89.14 \pm 6.09^{\mathrm{c}}$ & $4.76 \pm 0.42^{\mathrm{b}}$ & $0.60 \pm 0.15^{\mathrm{a}}$ & $0.26 \pm 0.09^{\mathrm{a}}$ \\
Low-dose EFM & 0 & $306.9 \pm 14.08^{\mathrm{a}}$ & $135.71 \pm 6.22^{\mathrm{a}}$ & $4.83 \pm 0.22^{\mathrm{ab}}$ & $0.55 \pm 0.12^{\mathrm{a}}$ & $0.15 \pm 0.05^{\mathrm{a}}$ \\
Medium-dose EFM & 0 & $310.3 \pm 12.51^{\mathrm{a}}$ & $138.02 \pm 5.65^{\mathrm{a}}$ & $4.44 \pm 0.18^{\mathrm{b}}$ & $0.54 \pm 0.12^{\mathrm{a}}$ & $0.17 \pm 0.01^{\mathrm{a}}$ \\
High-dose EFM & 0 & $321.8 \pm 15.58^{\mathrm{a}}$ & $141.68 \pm 7.09^{\mathrm{a}}$ & $4.31 \pm 0.33^{\mathrm{b}}$ & $0.61 \pm 0.08^{\mathrm{a}}$ & $0.19 \pm 0.08^{\mathrm{a}}$ \\
\hline
\end{tabular}


control group $(P<0.05)$. However, the liver coefficients of the medium- and high-dose EFM groups were significantly lower than that of the HFHS model group $(P<$ 0.05). As the liver coefficient reflects the degree of liver enlargement to a certain extent, these results indicate that medium- and high-dose EFM intervention provides certain inhibitory effects on the development of fatty liver in the experimental animals. Differences in kidney and spleen coefficients among the various groups were not significant $(P>0.05)$.

\section{Influence of EFM on fasting blood glucose and glucose tolerance levels of SD rats}

The diagnostic criterion for diabetes in experimental animals is a fasting blood glucose level of $>7.0 \mathrm{mmol} / \mathrm{L}$ (Yamanouchi et al., 2017). Figure 1 shows that the fasting blood glucose level in the serum of rats belonging to the HFHS model group was $7.55 \pm 0.48 \mathrm{mmol} / \mathrm{L}$, which was significantly higher than that of the normal control group $(4.86 \pm 0.53 \mathrm{mmol} / \mathrm{L})$. In addition, five out of eight rats of the HFHS model group had a fasting blood glucose level of $>7.0 \mathrm{mmol} / \mathrm{L}$, which indicates the successful construction of the HFHS diet-induced animal model of diabetes. From the dynamic monitoring of the blood glucose levels of the rats through the collection of tail vein blood samples at 0, 15, 30, 60 and $120 \mathrm{~min}$ after the intraperitoneal injection of glucose, it can be seen that the fasting blood glucose level rapidly increased to a peak value 0-15 min after glucose

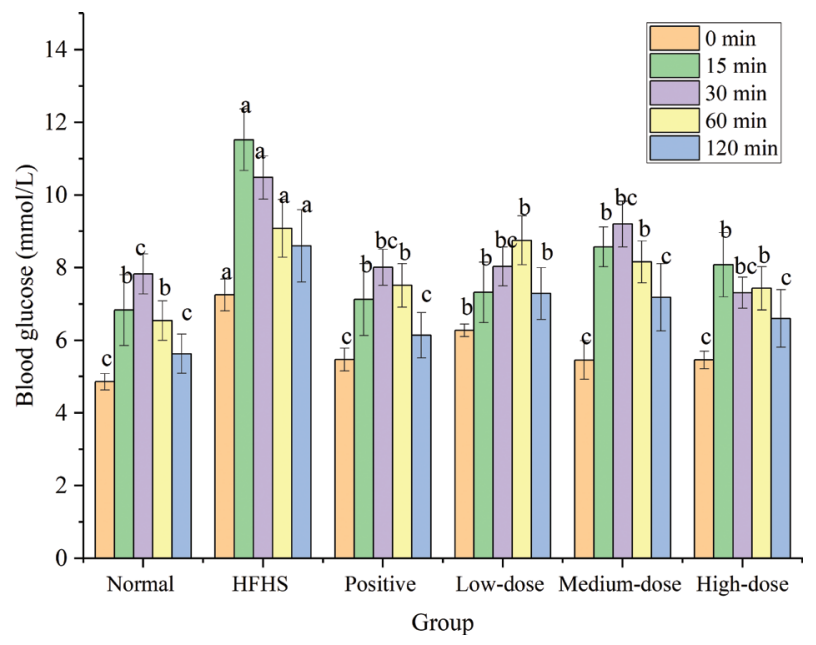

Figure 1. Influence of expanded flaxseed meal on the fasting blood glucose and glucose tolerance levels of SpragueDawley rats.

${ }^{a}$ Normal, HFHS, positive, low-dose, medium-dose, high-dose, respectively, represented the tissues of the normal control, HFHS model, positive control, low-dose EFM, medium-dose EFM and high-dose EFM groups.

${ }^{b}$ Different letters mean significant difference at the $5 \%$ level. injection and gradually decreased with time. In particular, the fasting blood glucose levels of the HFHS model group were always higher than that of other groups at all time points of the glucose tolerance experiment. A negative correlation existed between the fasting blood glucose level and EFM dose in the low-, medium- and high-dose EFM groups $(P<0.05)$. It was also observed that one rat in the low-dose EFM group had a fasting glucose level of $>7.0 \mathrm{mmol} / \mathrm{L}$. The experimental results indicated that EFM had a significant inhibitory effect on blood glucose elevation. However, as the activity of low-dose EFM in inhibiting blood glucose elevation was relatively low, an appropriate dose level is required to achieve sufficient inhibitory activity.

\section{Influence of EFM on $\mathrm{HbA1c}$ levels and hepatic glycogen concentrations in the liver tissue of SD rats}

HbA1c is formed through the linkage of haemoglobin in erythrocytes with glucose in the blood. Therefore, a higher HbA1c level corresponds to a greater amount of linkages between blood glucose and haemoglobin, and is indicative of a more severe diabetic condition (Yamane et al., 2019). HbA1c is of great significance in the monitoring of diabetes as it is positively correlated to blood glucose elevation (Yamane et al., 2019). Due to the slow formation process of HbA1c, temporary increases in blood glucose levels will not result in an elevated HbA1c level. However, as HbA1c does not degrade easily upon formation, the HbA1c level is unable to reflect short-term fluctuations in blood glucose levels, but provides a good indication of the adequacy of long-term blood glucose control and the average blood glucose level Yu (Koga et al., 2019). Figure 2 shows that the elevation of HbA1c concentration was significantly inhibited in both the medium- and high-dose EFM groups compared with the positive treatment control group $(P<0.05)$. The blood glucose level of an organism is maintained within a relatively stable range mainly through the regulatory effects of the nervous system, liver and hormones (Yu et al., 2019). As hepatic glycogen synthesis is one of the blood glucose consumption pathways, hepatic glycogen concentration is negatively correlated with blood glucose levels, that is, blood glucose levels decrease when hepatic glycogen concentrations increase and vice versa. In the present study, the HFHS model group had the lowest hepatic glycogen concentration of $11.82 \mathrm{mg} / \mathrm{g}$ liver tissue. Compared with the HFHS model group, the medium-dose EFM, high-dose EFM and positive control groups had significantly higher hepatic glycogen concentrations $(P<0.05)$. Therefore, it can be deduced that EFM possesses the ability to increase hepatic glycogen synthesis and reduce hepatic glycogen degradation. 
Influence of EFM on serum TG, TC, HDL-C and LDL-C concentrations, atherogenic index and anti-atherogenic index of SD rats

From Table 3, it can be seen that a long-term HFHS diet led to lipid metabolism disorders in the experimental animals, which resulted in a significant increase in the serum TG and TC contents of the rats $(P<0.05)$. Compared with the HFHS model group, EFM intervention in HFHS feed provided significant inhibitory effects on the elevation of blood lipid levels in the animals $(P<0.05)$. A dose-effect relationship was found between

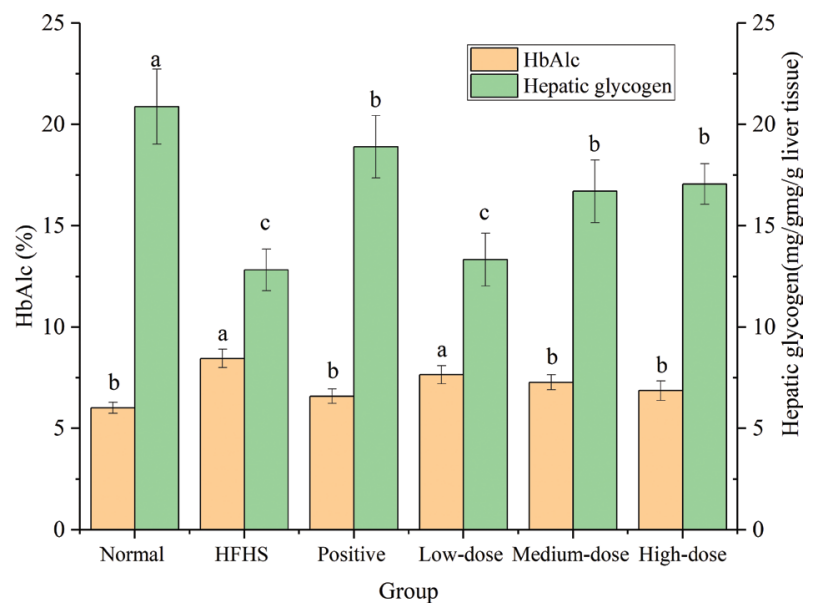

Figure 2. Influence of expanded flaxseed meal on $\mathrm{HbA1c}$ levels and hepatic glycogen concentrations in liver tissue of Sprague-Dawley rats.

${ }^{a}$ Normal, HFHS, positive, low-dose, medium-dose, high-dose, respectively, represented the tissues of the normal control, HFHS model, positive control, low-dose EFM, medium-dose EFM and high-dose EFM groups.

${ }^{b} \mathrm{HbA1c}$, glycated haemoglobin.

'Different letters mean significant difference at the $5 \%$ level. the EFM dosage and blood lipid levels, with higher EFM doses associated with lower plasma TG, TC and LDL-C concentrations, whereas the influence of EFM dosage on HDL-C concentration was not consistent. Clinical studies have shown that the atherogenic index (AI) is indicative of the comprehensive effects of blood lipid levels on atherosclerosis (Fernández-Macías et al., 2019). In general, a lower AI value represents a lower probability of the onset of atherosclerosis, while a higher AI value indicates a higher LDL-C and lower HDL-C concentration in the blood, which increases the risk of atherosclerosis. From the experimental data, it can be seen that the anti-atherogenic index (AAI) values of the positive treatment control and EFM dose groups were higher than that of the HFHS model group $(P<0.05)$, with a doseeffect relationship existing between the EFM dosage and AAI value; the AAI value of the low-dose EFM group was significantly different from that of the medium-and high-dose EFM groups $(P<0.05)$. Based on the results described above, it can be deduced that the intake of EFM at a certain dosage effectively inhibited the onset of atherosclerosis and reduced the risk of atherosclerosis brought about by calorie-rich diets.

\section{The influence of EFM on plasma antioxidant status and lipid peroxidation in SD rats}

SOD and GSH-Px are key antioxidant enzymes; SOD converts superoxide to hydrogen peroxide and GSH-Px metabolises the hydrogen peroxide converted by SOD into water (Bela et al., 2015; Sannasimuthu et al., 2018). GSH is a non-enzymatic antioxidant, which can directly react with free radicals or serve as an electron donor during the reduction of peroxides by GSH-Px (Min et al., 2018). The EFM used in the present study contained close to $40.7 \%$ of flaxseed oil, which is easily oxidised. Therefore, besides possessing antioxidant activity, EFM may even result in lipid peroxidation. Hence, the

Table 3. Influence of expanded flaxseed meal on serum total triglyceride, total cholesterol, high-density lipoprotein cholesterol and low-density lipoprotein cholesterol concentrations, atherogenic index and anti-atherogenic index of Sprague-Dawley rats.

\begin{tabular}{|c|c|c|c|c|c|c|}
\hline Group & TG & $\mathrm{TC}$ & HDL-C & LDL-C & Al & AAl \\
\hline Normal control & $1.10 \pm 0.05^{b}$ & $1.67 \pm 0.14^{b}$ & $0.78 \pm 0.06^{\mathrm{a}}$ & $0.29 \pm 0.03^{c}$ & $1.25 \pm 0.12^{d}$ & $0.44 \pm 0.02^{\mathrm{a}}$ \\
\hline HFHS model & $1.81 \pm 0.10^{\mathrm{a}}$ & $2.41 \pm 0.20^{\mathrm{a}}$ & $0.58 \pm 0.04^{c}$ & $0.82 \pm 0.08^{a}$ & $3.15 \pm 0.18^{a}$ & $0.24 \pm 0.08^{d}$ \\
\hline Positive control & $1.08 \pm 0.06^{b}$ & $1.98 \pm 0.08^{b}$ & $0.74 \pm 0.03^{\mathrm{ab}}$ & $0.36 \pm 0.02^{c}$ & $1.66 \pm 0.18^{c}$ & $0.38 \pm 0.03^{b}$ \\
\hline Low-dose EFM & $1.28 \pm 0.07^{\mathrm{b}}$ & $1.86 \pm 0.12^{b}$ & $0.68 \pm 0.06^{\mathrm{ab}}$ & $0.56 \pm 0.05^{b}$ & $2.07 \pm 0.38^{b}$ & $0.30 \pm 0.02^{c}$ \\
\hline Medium-dose EFM & $1.22 \pm 0.08^{b}$ & $1.76 \pm 0.15^{b}$ & $0.62 \pm 0.05^{b}$ & $0.40 \pm 0.06^{c}$ & $1.75 \pm 0.11^{\mathrm{bc}}$ & $0.36 \pm 0.02^{b}$ \\
\hline High-dose EFM & $1.17 \pm 0.08^{b}$ & $1.74 \pm 0.10^{b}$ & $0.70 \pm 0.03^{\mathrm{ab}}$ & $0.33 \pm 0.02^{c}$ & $1.61 \pm 0.09^{c}$ & $0.38 \pm 0.05^{b}$ \\
\hline
\end{tabular}


Table 4. Influence of expanded flaxseed meal on plasma antioxidant status and lipid peroxidation in Sprague-Dawley rats.

\begin{tabular}{|c|c|c|c|c|c|}
\hline Group & GSH-Px (U/mL) & $\mathrm{SOD}(\mathrm{U} / \mathrm{mL})$ & CAT (U/mL) & $\mathrm{GSH}(\mathrm{mg} / \mathrm{L})$ & MDA (nmol/mL) \\
\hline Normal control & $1189.75 \pm 35.82^{\mathrm{a}}$ & $102.24 \pm 1.25^{a}$ & $7.80 \pm 0.25^{\mathrm{a}}$ & $12.88 \pm 0.49^{b}$ & $1.46 \pm 0.09^{c}$ \\
\hline HFHS model & $810.30 \pm 25.48^{d}$ & $85.27 \pm 1.68^{b}$ & $3.48 \pm 0.24^{d}$ & $10.48 \pm 0.26^{c}$ & $3.33 \pm 0.15^{\mathrm{a}}$ \\
\hline Positive control & $1148.67 \pm 37.68^{a b}$ & $100.96 \pm 2.25^{\mathrm{a}}$ & $4.69 \pm 0.19^{c}$ & $15.87 \pm 0.68^{\mathrm{a}}$ & $2.16 \pm 0.12^{b}$ \\
\hline Low-dose EFM & $897.46 \pm 26.36^{c}$ & $99.91 \pm 1.98^{\mathrm{a}}$ & $4.88 \pm 0.17^{c}$ & $13.37 \pm 0.75^{b}$ & $2.04 \pm 0.16^{b}$ \\
\hline Medium-dose EFM & $1087.29 \pm 40.25^{b}$ & $98.61 \pm 3.05^{\mathrm{a}}$ & $5.19 \pm 0.25^{c}$ & $15.27 \pm 0.66^{a}$ & $1.65 \pm 0.08^{c}$ \\
\hline High-dose EFM & $1169.22 \pm 42.28^{\mathrm{ab}}$ & $100.70 \pm 1.29^{a}$ & $5.80 \pm 0.24^{b}$ & $15.67 \pm 0.55^{\mathrm{a}}$ & $1.62 \pm 0.13^{c}$ \\
\hline
\end{tabular}

${ }^{a} \mathrm{GSH}-\mathrm{Px}, \mathrm{SOD}, \mathrm{CAT}, \mathrm{GSH}$ and MDA indicate the tissues of the glutathione peroxidase, superoxide dismutase, catalase, glutathione and malondialdehyde, respectively.

${ }^{b}$ Different letters mean significant difference at the $5 \%$ level.

EFM, expanded flaxseed meal; HFHS, high-fat, high-sugar; CSH, SOD, superoxide dismutase; CAT, catalase; GSH, glutathione; MDA, malondialdehyde

determination of the influence of EFM intake on plasma antioxidant status and lipid peroxidation in SD rats is of great significance. From Table 4, it can be seen that plasma antioxidant activity increased, and lipid peroxidation reduced significantly $(P<0.05)$ in the various EFM dose groups compared with the HFHS model group. Compared with the positive control group, feeding rats with feed containing a medium or high dose of EFM for 8 weeks also significantly increased the GSH-Px and GSH levels in the plasma $(P>0.05)$. In addition, dose-effect relationships of various degrees existed between EFM and the antioxidant status indicators (except for SOD). The MDA level is commonly used as a marker for the assessment of lipid peroxidation effects (Tsikas, 2017). Compared with the positive treatment control group, MDA concentration decreased significantly in the medium- and high-dose EFM groups $(P<0.05)$. The indicator values obtained in this study suggest that the intake of EFM provided good antioxidant effects in the plasma of SD rats on a HFHS diet.

\section{Influence of EFM on the hepatic and histological morphology of SD rats}

The liver tissue sections of SD rats were subjected to paraffin embedding and H\&E staining. Figure 3 shows the histological morphologies of the liver tissues of SD rats of the various experimental groups at $400 \times$ magnification. As shown in Figure 3A and 3B, the hepatic cells of the normal control group exhibited a loose and uniform arrangement with relatively intact structures and small intercellular spaces, while the HFHS model group had a significantly enlarged liver with a disorderly arrangement of hepatic cells and large lipid droplets that were densely accumulated into vacuoles of various sizes. With the aggravation of steatosis, these vacuoles may gradually expand and become distributed in the entire cytoplasm, thereby increasing hepatic damage (Baboota et al., 2018). Compared with the HFHS model group, the positive control, medium-dose EFM and high-dose EFM groups showed improvements in hepatic histological morphology, with the vacuoles and lipid droplets being significantly smaller and less densely arranged. These results indicate that EFM intake enabled an improvement in the size and density of lipid droplets in the liver tissues of SD rats and inhibited the onset of high-fat diet-induced fatty liver.

\section{Conclusions}

In this study, expanded flaxseed meal (EFM) produced from the pre-treatment of flaxseed using the steam explosion technique was added to the feed of experimental animals. Normal growth and development were observed in the animals, which demonstrates the safety of EFM within the dose range adopted in the study. The fasting blood glucose level and HbA1c concentration of the HFHS model group increased significantly compared with the normal control group $(P<0.05)$, indicating the successful establishment of a HFHS diet-induced hyperglycaemic diabetes rat model. With the addition of EFM in the HFHS feed, the fasting blood glucose levels and HbA1c concentrations of the medium- and highdose EFM groups were significantly lower than that of the HFHS model group $(P<0.05)$. In addition, changes in the fasting blood glucose level and HbA1c concentration across the various EFM dose groups indicated the existence of a dose-effect relationship between EFM and the inhibition of blood glucose elevation. The blood glucose levels and HbA1c concentrations of the medium- and high-dose EFM groups were not significantly different from that of the positive treatment control group $(P<0.05)$, which had received acarbose, a commonly used medication for managing diabetes. The experimental results also showed that EFM, not only significantly reduced the blood lipid levels of SD rats on a HFHS diet, but also significantly increased the plasma antioxidant capability and reduced the level of 

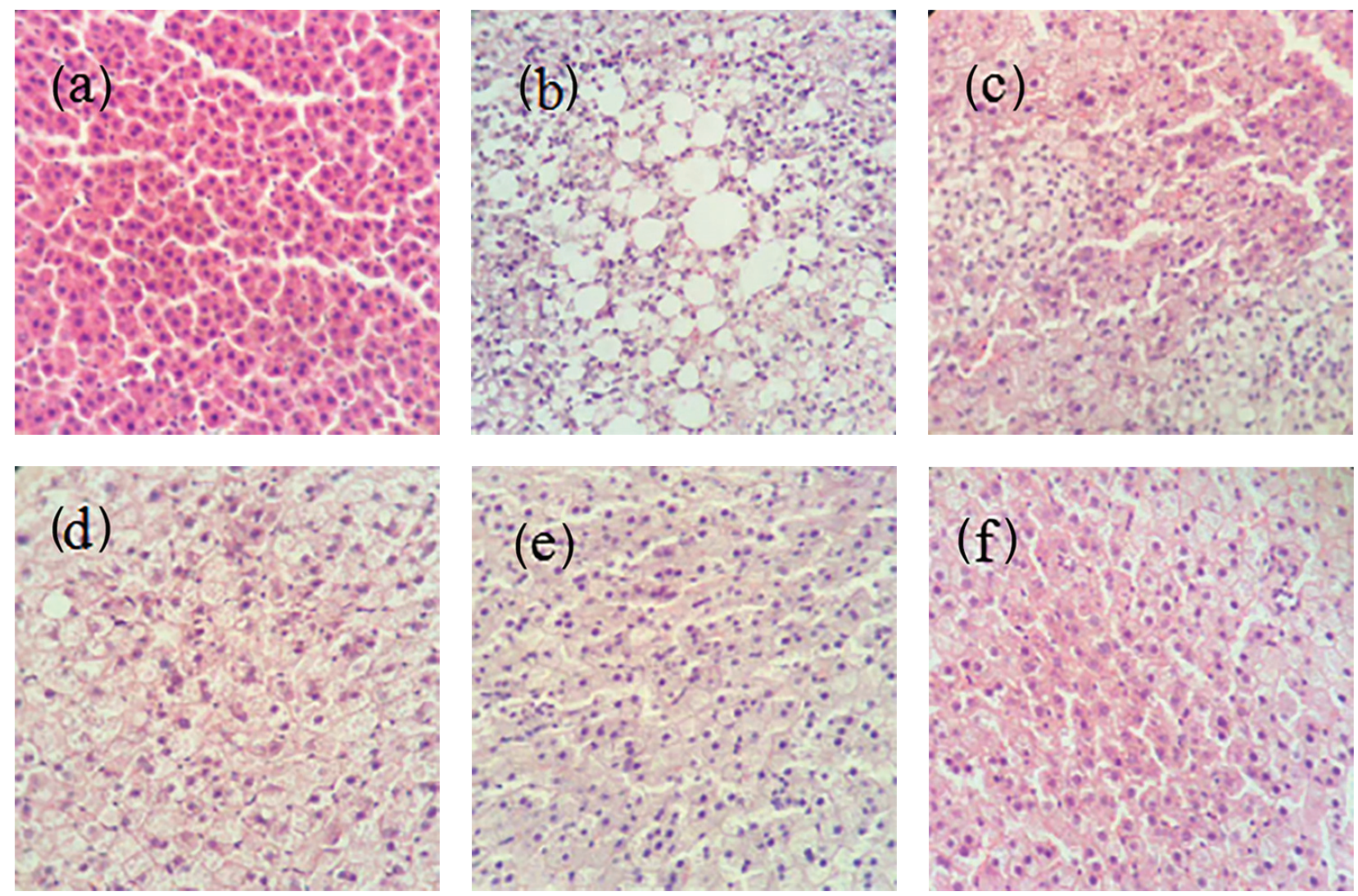

Figure 3. The influence of expanded flaxseed meal on hepatic histological morphology of Sprague-Dawley rats.

(A-F indicate the tissues of the normal control, HFHS model, positive control, low-dose EFM, medium-dose EFM, and high-dose EFM groups, respectively).

lipid peroxidation products in the plasma. Furthermore, the hepatic histological morphologies of the various groups indicated that EFM enabled the improvement of the size and density of lipid droplets in the liver tissues of SD rats and inhibited the onset of high-fat diet-induced fatty liver.

\section{Acknowledgement}

The authors would like to thank three anonymous reviewers and the editors for their comments.

\section{Conflicts of Interest}

The authors declare no conflicts of interest with respect to research, authorship and/or publication of this article.

\section{Compliance with ethical standards}

All experimental protocols were approved by the Ethical Committee of the Faculty of Veterinary Medicine, Huazhong Agricultural University (permit number: SYXK-ER-2013-0044). The study was performed in strict accordance with the recommendations of the Guide for the Care and Use of Laboratory Animals in China.

\section{Funding}

This study was partially funded by Earmarked Fund for China Agriculture Research System (CARS14); Agricultural Science and Technology Innovation Project of Chinese Academy of AgricSciences (CAAS-ASTIP-2013-0CRI).

\section{References}

Alberdicedeño, J., Ibargoitia, M.L., Cristillo, G., Sopelana, P. and Guillén, M.D., 2017. A new methodology capable of characterizing most volatile and less volatile minor edible oils components in a single chromatographic run without solvents or reagents detection of new components. Food Chemistry 221: 1135-1144. https://doi.org/10.1016/j.foodchem.2016.11.046.

Baboota, R.K., Khare, P., Mangal, P., Singh, D.P., Bhutani, K.K., Kondepudi, K.K., Kaur, J. and Bishnoi, M., 2018. Dihydrocapsiate supplementation prevented high-fat diet-induced adiposity, 
hepatic steatosis, glucose intolerance, and gut morphological alterations in mice. Nutrition Research 51: 40-56. https://doi. org/10.1016/j.nutres.2017.11.006.

Bekhit, A.E.-D.A., Shavandi, A., Jodjaja, T., Birch, J., Teh, S., Mohamed Ahmed, I.A., Al-Juhaimi, F.Y., Saeedi, P. and Bekhit, A.A., 2018. Flaxseed: composition, detoxification, utilization, and opportunities. Biocatalysis and Agricultural Biotechnology 13: 129-152. https://doi.org/10.1016/j.bcab.2017.11.017

Bela, K., Horváth, E., Gallé, Á., Szabados, L., Tari, I. and Csiszár, J., 2015. Plant glutathione peroxidases: emerging role of the antioxidant enzymes in plant development and stress responses. Journal of Plant Physiology 176: 192-201. https://doi.org/10.1016/j. jplph.2014.12.014

Cao, W.W., Huang, Q.D., Tian, G.J. and Deng, Q.C., 2016. Effects of microwave pretreatment on the content of cyanogenic glucosides in pressed flaxseed cakes and the quality of pressed oil. Science and Technology of Food Industry 37: 134-138.

Chirino-Galindo, G., Barrera-Argüelles, J.-I., Trejo-González, N.-L., Mejía-Zepeda, R. and Palomar-Morales, M., 2017. Biphasic effect of alpha-linolenic acid on glucose-induced dysmorphogenesis and lipoperoxidation in whole rat embryo in culture. Biochemical and Biophysical Research Communications 484: 878-883. https://doi. org/10.1016/j.bbrc.2017.02.011

Chu, M., Hai, W., Zhang, Z., Wo, F., Wu, Q., Zhang, Z., Shao, Y., Zhang, D., Jin, L. and Shi, D., 2016. Melanin nanoparticles derived from a homology of medicine and food for sentinel lymph node mapping and photothermal in vivo cancer therapy. Biomaterials 91: 182-199. https://doi.org/10.1016/j.biomaterials.2016.03.018

Cressey, P. and Reeve, J., 2019. Metabolism of cyanogenic glycosides: a review. Food and Chemical Toxicology 125: 225-232. https:// doi.org/10.1016/j.fct.2019.01.002

Edrosolam, M.G. and Shand, P.J., 2014. Utilization of flaxseed meal and microbial transglutaminase in processing of low-salt, low-fat bologna. Meat Science 96: 120-121. https://doi.org/10.1016/j. meatsci.2013.05.049

Fernández-Macías, J., Ochoa-Martínez, A., Varela-Silva, J. and Pérez-Maldonado, I., 2019. Atherogenic Index of Plasma: Novel Predictive Biomarker for Cardiovascular Illnesses. Archives of Medical Research 50: 285-294. https://doi.org/10.1016/j. arcmed.2019.08.009

Ganorkar, P.M., Patel, J.M., Shah, V. and Rangrej, V.V., 2016. Defatted flaxseed meal incorporated corn-rice flour blend based extruded product by response surface methodology. Journal of Food Science and Technology 53: 1867-1877. https://doi.org/10.1007/ s13197-015-2134-3

Han, H., Ma, H., Rong, S., Chen, L., Shan, Z., Xu, J., Zhang, Y. and Liu, L., 2015. Flaxseed oil containing flaxseed oil ester of plant sterol attenuates high-fat diet-induced hepatic steatosis in apolipoprotein-E knockout mice. Journal of Functional Foods 13: 169-182. https://doi.org/10.1016/j.jff.2014.12.046

Han, H., Qiu, F., Zhao, H., Tang, H., Li, X. and Shi, D., 2018. Dietary flaxseed oil improved western-type diet-induced atherosclerosis in apolipoprotein-E knockout mice. Journal of Functional Foods 40: 417-425. https://doi.org/10.1016/j.jff.2017.11.031

Koga, M., Inada, S., Shibata, M., Ijima, H., Jinnouchi, H., Ono, Y., Iwasaka, T., Tokuhiro, S., Matsumura, Y., Matsui, H. and
Okumiya, T., 2019. HbA1c adjusted by erythrocyte creatine is a useful glycemic control indicator in patients with hemolysis. Clinical Biochemistry 73: 77-81. https://doi.org/10.1016/j. clinbiochem.2019.08.004

Li, P.P., Huang, Q.D., Xu, J.Q., Zhang, Y.L., Ma, C.C. and Deng, Q.C., 2018. Effect of Partially Defatted Flaxseed Meal on Blood Glucose in Rats Fed with High-Fat High-Sugar Diet. Food Science 39: 183-187.

Li, B., Yang, W., Nie, Y., Kang, F., Goff, H.D. and Cui, S.W., 2019. Effect of steam explosion on dietary fiber, polysaccharide, protein and physicochemical properties of okara. Food Hydrocolloids 94: 48-56. https://doi.org/10.1016/j.foodhyd.2019.02.042

Liu, J. and Ma, D.W.L., 2014. The role of $n-3$ polyunsaturated fatty acids in the prevention and treatment of breast cancer. Nutrients 6: 5184-5223. https://doi.org/10.3390/nu6115184.

Luo, J., Li, Y., Mai, Y., Gao, L., Ou, S., Wang, Y., Liu, L. and Peng, X., 2018. Flaxseed gum reduces body weight by regulating gut microbiota. Journal of Functional Foods 47: 136-142. https://doi. org/10.1016/j.jff.2018.05.042

Min, Y., Niu, Z., Sun, T., Wang, Z., Jiao, P., Zi, B., Chen, P., Tian, D. and Liu, F., 2018. Vitamin E and vitamin C supplementation improves antioxidant status and immune function in oxidative-stressed breeder roosters by up-regulating expression of GSH-Px gene. Poultry Science 97: 1238-1244. https://doi. org/10.3382/ps/pex4.17

Sannasimuthu, A., Kumaresan, V., Pasupuleti, M., Paray, B.A., Al-Sadoon, M.K. and Arockiaraj, J., 2018. Radical scavenging property of a novel peptide derived from C-terminal SOD domain of superoxide dismutase enzyme in Arthrospira platensis. Algal Research 35: 519-529. https://doi.org/10.1016/j. algal.2018.09.028

Shi, Q., Li, Y., Li, Y., Cheng, Y. and Zhu, W., 2019. Effects of steam explosion on lignocellulosic degradation of, and methane production from, corn stover by a co-cultured anaerobic fungus and methanogen. Bioresource Technology 290: 121796. https://doi. org/10.1016/j.biortech.2019.121796

Song, D.-X. and Jiang, J.-G., 2017. Hypolipidemic components from medicine food homology species used in China: pharmacological and health effects. Archives of Medical Research 48: 569-581. https://doi.org/10.1016/j.arcmed.2018.01.004

Tsikas, D., 2017. Assessment of lipid peroxidation by measuring malondialdehyde (MDA) and relatives in biological samples: analytical and biological challenges. Analytical Biochemistry 524: 13-30. https://doi.org/10.1016/j.ab.2016. 10.021

Wang, H., Hong, T., Li, N., Zang, B. and Wu, X., 2018. Soluble dietary fiber improves energy homeostasis in obese mice by remodeling the gut microbiota. Biochemical and Biophysical Research Communications 498: 146-151. https://doi.org/10.1016/j. bbrc.2018.02.017

Wang, Y., Fofana, B., Roy, M., Ghose, K., Yao, X.-H., Nixon, M.-S., Nair, S. and Nyomba, G.B.L., 2015. Flaxseed lignan secoisolariciresinol diglucoside improves insulin sensitivity through upregulation of GLUT4 expression in diet-induced obese mice. Journal of Functional Foods 18: 1-9. https://doi.org/10.1016/j. jff.2015.06.053 
Yamane, T., Imai, M., Handa, S., Yamada, K., Sakamoto, T., Ishida, T., Inui, H., Yamamoto, Y., Nakagaki, T. and Nakano, Y., 2019. Reduction of blood glucose and HbA1c levels by cyanidin 3,5-diglucoside in KKAy mice. Journal of Functional Foods 58: 21-26. https://doi.org/10.1016/j.jff.2019.04.038

Yamanouchi, M., Skupien, J., Niewczas, M.A., Smiles, A.M., Doria, A., Stanton, R.C., Galecki, A.T., Duffin, K.L., Pullen, N., Breyer, M.D., Bonventre, J.V., Warram, J.H. and Krolewski, A.S., 2017. Improved clinical trial enrollment criterion to identify patients with diabetes at risk of end-stage renal disease. Kidney International 92: 258-266. https://doi.org/10.1016/j.kint.2017.02.010

Yang, H.Z., Sun, W.J. and Zhong, Y.C., 2008. Study on four detoxification technologies of flaxseed. Food Science 9: 216-219.

Yu, H., Wang, J., Shrestha, Y., Hu, Y., Ma, Y., Ren, L., Zhang, J. and Li, J., 2019. Importance of early elevated maternal HbA1c levels in identifying adverse fetal and neonatal events. Placenta 86: 28-34. https://doi.org/10.1016/j. placenta.2019.07.008

Zang, X.X., Wei, X.S., Chen, P. Zhang, Y. Huang, H.F. and Deng, Q.C., 2017. Lignan content and its hydrolysates of flaxseeds in different cultivars. Chinese Journal of Oil Crop Sciences 39: 253-259.

Zhang, S., Zheng, L., Zheng, X., Ai, B., Yang, Y., Pan, Y. and Sheng, Z., 2019. Effect of steam explosion treatments on the functional properties and structure of camellia (Camellia oleifera Abel.) seed cake protein. Food Hydrocolloids 93: 189-197. https://doi. org/10.1016/j.foodhyd.2019.02.017

Zhao, M., Bergaentzlé, M., Flieller, A. and Marchioni, E., 2019. Development and validation of an ultra-high performance liquid chromatography-high resolution mass spectrometry method for simultaneous quantification of cyanogenic glycosides and secoisolariciresinol diglucoside in flaxseed (Linum usitatissimum L.). Journal of Chromatography. A 1601: 214-223. https://doi.org/ 10.1016/j.chroma.2019.04.072 